\title{
INTERPOLATION OF RADIOACTIVITY DATA USING REGULARIZED SPLINE WITH TENSION
}

\author{
Jaroslav Hofierka \\ Department of Geography and Regional Development, Faculty of Humanities and \\ Natural Sciences, University of Prešov, Prešov (Slovak Republic) \\ Correspondence to: hofierka@fhpv.unipo.sk
}

\begin{abstract}
Regularized Spline with Tension was used to interpolate two data sets representing radioactivity measurements at 200 locations. A cross-validation analysis showed that the size of the training data sets was too low to find optimal parameters using the cross-validation procedure. The resulting surfaces were strongly smoothed and less realistic than expected. Therefore empirical interpolation parameters were used to interpolate the data. Despite the fact that this empirical selection did not produced interpolation results with a lower overall predictive error, it preserved better local fluctuations and anomalies of the phenomenon. The detection of these features is important in radioactivity monitoring and emergency situations. The poor reliability of cross-validation was also confirmed by evaluation data set. It was concluded that the optimization of interpolation parameters cannot rely on cross-validation when the modeled phenomenon is not sufficiently sampled. The sampling density should be sufficient to represent spatial variations of the phenomenon and, at the same time, allow the optimization of interpolation parameters using automated procedures.
\end{abstract}

\section{INTRODUCTION}

Many environmental variables are measured in a limited number of locations. The purpose of spatial interpolation is to estimate values of the measured phenomenon in areas between measured data points and, at the same time, pass through the given points as close as possible. There are many interpolation methods that can be used for this task (see e.g. Burrough 1986, Franke 1982, Cressie 1993, Mitas and Mitasova 1999). They differ on additional interpolation conditions that define the character of the method. Typical examples are conditions based on geostatistical concepts (Kriging), locality (nearest neighbour), smoothness and tension (splines), or ad hoc functional forms (polynomials, multi-quadrics). Choice of the additional condition depends on the character of the modeled phenomenon and the type of application (Mitas and Mitasova 1999). However, it may be difficult to find a method which satisfies demands on interpolation accuracy, computational efficiency, robustness and flexibility. Many modeled phenomena are very complex and often poorly sampled. Therefore, the selection of an adequate method with appropriate parameters for a particular application is crucial. The use of inappropriate method or parameters can result in a distorted model of spatial distribution, leading to potentially wrong decisions. 
The Spatial Interpolation Comparison 2004 (SIC2004) is focused on many of above aspects. A spatial distribution of natural ambient radioactivity has a very complex character. While the general pattern of the radioactivity does not change over a long time, it may be abruptly changed by events like Chernobyl nuclear power plant accident or terrorist attack. In such situations a quick spatial representation of radioactivity distribution is crucial for many decisions. Certainly, spatial interpolation plays an important role in this task. The goal is to get interpolation results as fast as possible and, at the same time, sufficiently reliable.

In this article, the bivariate Regularized Spline with Tension (RST) implemented in GRASS GIS as s.surf.rst command is used to make the required estimates of radioactivity at 808 locations, based on "training" sets of radioactivity measurements at 200 locations throughout Germany.

The presented approach consists of several parts. First, the optimum set of parameters for the RST method and provided data is selected using cross-validation and visual evaluation of the interpolation results for the training data sets. Then the estimates for 2 test data sets are made using a parametrized interpolator. The obtained interpolation results are verified using evaluation data set and discussed in the last part.

\section{INTERPOLATION BY REGULARIZED SPLINE WITH TENSION}

The Regularized Spline with Tension (RST) is an interpolation function based on variational approach to the interpolation problem (Mitas and Mitasova 1999). The method belongs to the wide group of the radial basis functions that differ according to the chosen basis function. The RST method fulfills two imposed interpolation conditions. The interpolation function should pass through the data points and, at the same time, it should be as smooth as possible. These conditions are expressed by minimization of deviations from the measured points and its smoothness seminorm:

$$
\sum_{j=1}^{N} \mid p_{j}-S\left(\mathbf{x}_{j}\right)^{2} w_{j}+w_{0} I(S)=\text { minimum }
$$

where $S(\mathbf{x})$ is the RST function, $p_{j}$ are the values measured at discrete points $\mathbf{x}_{j}=\left(x_{j}, y_{j}\right), j=1, \ldots, N$ within a region of a 2 -dimensional space, $w_{j}, w_{0}$ are positive weighting factors and $I(S)$ is the measure of smoothness (smooth seminorm or roughness penalty). For $w_{j} / w_{0}=0$ the function $S(\mathbf{x})$ passes exactly through the given points. The general solution of the minimization problem given by equation (1) can be expressed as a sum of two components (Talmi and Gilat 1977):

$$
S(\mathbf{x})=T(\mathbf{x})+\sum_{j=1}^{N} \lambda_{j} R\left(\mathbf{x}, \mathbf{x}_{j}\right)
$$

where $T(\mathbf{x})$ is a "trend" function and $R\left(\mathbf{x}, \mathbf{x}_{j}\right)$ is a radial basis function with an explicit form depending on the choice of the $I(S)$. The smoothness seminorm $I(S)$ for the RST method has been designed to synthesize in a single function properties of several previously known splines, such as Thin Plate Spline (Duchon 1976), Thin Plate Spline 
with Tension (Franke 1985, Mitas and Mitasova 1988), Regularized Thin Plate Spline (Mitas and Mitasova 1988). These desired properties include an explicit form, multivariate formulation, smooth derivatives of higher orders, variational freedom through tension and anisotropy.

The seminorm which fulfills these requirements includes derivatives of all orders with weights rapidly decreasing with the increasing derivative order. For $d=2$ it has the following form (Hofierka et al. 2002):

$$
I^{2}(S)=\sum_{\alpha} B_{\alpha} \iint_{\Omega}\left[\frac{\partial^{|\alpha|}}{\partial x^{\alpha_{1}} \partial y^{\alpha_{2}}} S(\mathbf{x})\right]^{2} d x d y
$$

where $\alpha=\left(\alpha_{1}, \alpha_{2}\right)$ is a multiindex $\left(\alpha_{1}=0,1,2, \ldots, \alpha_{2}=0,1,2, \ldots\right)$, with $|\alpha|=\alpha_{1}+\alpha_{2}$ and $\left\{B_{\alpha}\right\}$ are nonnegative constants, in our case

$$
B_{\alpha}= \begin{cases}0, & |\alpha|=0 \\ \frac{\mid \alpha !}{\alpha_{1} ! \alpha \alpha_{2} !} \frac{1 .}{\varphi^{2|\alpha|}(|\alpha|-1) !}, & |\alpha|>0\end{cases}
$$

where $\varphi$ is a relative reciprocal weight of particular terms in the sum (generalized tension) which provides the control over the influence of derivatives of certain order on the resulting function.

In 2-D formulation, the RST function generally defined by (2) has the following explicit form (Mitasova et al. 1995):

$$
S(\mathbf{x})=a_{1}+\sum_{j=1}^{N} \lambda_{j}\left\{-\left[E_{1}(\rho)+\ln \rho+C_{E}\right]\right\},
$$

where $\rho=(\varphi r / 2)^{2}, r^{2}=\left(x-x_{j}\right)^{2}+\left(y-y_{j}\right)^{2}$ is the squared distance, $C_{E}=0.577215$.. is the Euler constant, $E_{1}($.$) is the exponential integral function (Abramowitz and Stegun$ 1964), and $\varphi$ is a generalized tension parameter which provides the control over the influence of derivatives of certain order on the resulting function.

\section{THE RST PARAMETERS}

The bivariate RST interpolation has been implemented in GRASS GIS version 5.4 as s.surf.rst and v.surf.rst commands and is widely used by the GRASS GIS users community (Neteler and Mitasova 2002). In these commands, the interpolation process is controled by a set of the following parameters:

- tension $\varphi$,

- smoothing $w$,

- anisotropy $(\theta, s)$, where $\theta, s$ are the rotation and scale, respectively,

- minimum and maximum distances between points. 
The tension $\varphi$, smoothing $w$ and anisotropy $(\theta, s)$ are internal RST parameters and control the character of the resulting surface. The parameters can be selected empirically, based on the knowledge of the modeled phenomenon, or automatically, by minimization of the predictive error estimated by a cross-validation procedure (Hofierka et al. 2002).

The tension parameter controls the behaviour of the resulting surface from a thin membrane to a stiff steel plate. The RST method is scale dependent and the tension works as a rescaling parameter (Neteler and Mitasova 2002). A high tension "increases the distances between the points" and reduces the range of impact of each point, low tension "decreases the distance" and the points influence each other over a longer range. The tension parameter plays a key role in areas with a steep change of modeled phenomenon where overshoots and undershoots of the interpolated surface may occur. Surface with tension set too high behaves like a membrane (rubber sheet stretched over the data points) with peak or pit in each given point and everywhere else the surface goes rapidly to trend. Surface with tension set too low behaves like a stiff steel plate and overshoots can appear in areas with rapid change of gradient.

Using the smoothing parameter $w$, the RST behaves like an approximation function, i.e. the resulting surface does not pass through the given points, but approximates the input values. This parameter is useful in modeling noisy data, where higher smoothing can filter out the noise, or alternatively, when a phenomenon needs to be modeled at a lower level of detail.

The anisotropy parameters $(\theta, s)$ can be used for interpolation of anisotropic data. The orientation of the perpendicular axes characterizing the anisotropy is defined by the rotation parameter $\theta$ and the scaling ratio of the perpendicular axes (a ratio of axes sizes) is defined by the scale parameter $S$. These parameters scale distances (i.e. the value of tension) in 2 perpendicular directions that should fit the spatial pattern of the anisotropic phenomenon.

Minimum and maximum distances between points control the number of points that are actually used in interpolation after reading the input data. The maximum distance can be used only for vector data in the GRASS GIS version 6.0.0. However, this parameter internally influences the effect of the tension, because the tension works as a distancescaling factor. Therefore, the tension can be set with or without normalization. The data density does not affect the normalized tension parameter. In this study we use only the normalized tension.

\section{USE OF PRIOR INFORMATION AND SELECTING THE PARAMETERS}

During the training phase, the participants were provided with a set of 10 files containing measurements at 200 locations for 10 randomly selected days. These data were the only information available prior to the test phase. The values and spatial pattern of measured values were very similar for all days. They did not contain any major anisotropic features that would require the use of anisotropy parameters. The only RST parameters that could be optimized were tension and smoothing.

The optimal RST parameters for training data were selected using a cross-validation (CV) procedure. The CV procedure is based on removing one input data point at a time, performing the interpolation for the location of the removed point using the remaining samples and calculating the residual between the actual value of the removed data point 
and its estimate. The procedure is repeated until every sample has been, in turn, removed. This form of CV is also known as the "leave-one-out" method (Hofierka et al. 2002). The CV procedure was iteratively performed for all reasonable combinations of the RST parameters with small incremental steps (tension $\varphi \in<10,200>$, and smoothing $w \in<0.1,2.0>$ ).

The CV procedure is especially suitable for relatively dense data sets, since removing points from already under-sampled areas can lead to misrepresentation of the surface to be interpolated (the surface is smoothed). Despite the wide use of this technique for assessing interpolation schemes, one should be aware of its shortcomings. Specifically, CV will usually overestimate the interpolation error because the estimate is being computed at a location where data are genuinely available. In addition, the computed surface and hence the cross-validated estimate may be altered by the removal of the point being cross-validated. In practice these issues are unavoidable but with increasing number of input data points they have less impact (Jeffrey et al. 2001).

The overall performance of the interpolator is then evaluated by statistical means such as the Root Mean of Squared Error, Mean Absolute Error (MAE) or Mean Error (ME). Low Root Mean Squared Error (RMSE) indicates an interpolator that is likely to give most reliable estimates in areas with no data. The minimum statistical errors calculated by CV can be used to find the optimum interpolation parameters (Mitasova et al. 1995), (Hofierka et al. 2002). However, Hutchinson (1998) has found that CV does not always represent a reliable estimate of the model error, especially when a short-range correlation in data is present.

Our CV analysis showed that optimal RST parameters found by minimization of the root mean square error (RMSE) of cross-validation are tension $=10$ and smoothing $=0.6$ for most provided training files. Obviously, the interpolated surfaces were very smooth and neglected local fluctuations and anomalies in data. This was also confirmed by a large RMSE for interpolation deviations at input points. As one of the goals of this SIC 2004 event was to handle anomalies, it was concluded that sampling density represented by provided data sets is very low and $\mathrm{CV}$ in this case cannot guarantee the optimal interpolation result.

Therefore we decided to use empirical, ad hoc RST parameters (tension $=40$ and smooth $=0.1$ ), which are default values for these parameters for GRASS RST commands. The selection of these parameters was based on visual inspection of obtained results during the training phase and our previous experience with similar data. It was obvious that these ad hoc parameters could not provide interpolation results with the lowest overall prediction error, but our intention was to preserve anomalies and local fluctuations in data that should have more importance in emergency situations.

\section{RESULTS AND DISCUSSION}

During the test phase the SIC 2004 participants were provided by 2 data sets each containing 200 points. The routine data set was similar to the training sets. The emergency data set contained a sharp spike in data values (Table 1).

Based on our previous premise that the minimization of $\mathrm{CV}$ error cannot provide optimal interpolation results in this case, we used the empirical, ad-hoc RST parameters selected in the training phase for both data sets. 
The accuracy of the interpolation results was verified using an evaluation data set which consisted of 808 observed points for both routine and simulated emergency data sets. The general statistics for interpolation with empirical parameters used in the test is showed in Table 1 and Figures 1 and 3. The statistics for interpolation with parameters found later by a CV procedure are shown in Table 2 and Figure 2 and 4. Clearly, the parameters found by $\mathrm{CV}$ smoothed out the resulting surface neglecting many spatial variations in data. The minimal estimated values are above the minimal values of the evaluation data set and maximal values are well-below the maximal values of the evaluation data set. The smoothed interpolation is also obvious from standard deviations which are very especially low for the emergency data set. In this respect, the empirical, ad hoc parameters used for the test produced better results for both test data sets. The negative minimal value of estimated values for the emergency data set is caused by interpolation undershoot. In practical applications, the negative values can be replaced by zeros, because the negative radioactivity is not meaningful. It should be noted that these negative values (undershoots) also influenced the overall statistical errors of interpolation.

\begin{tabular}{|lrrrrr|}
\hline $\mathbf{N}=\mathbf{8 0 8}$ & min. & max. & mean & median & std. dev. \\
\hline Observed (routine data set) & 57.00 & 180.00 & 98.02 & 98.80 & 20.01 \\
Estimates (routine data set) & 56.96 & 142.84 & 96.91 & 98.58 & 17.58 \\
\hline Observed (emergency data set) & 57.00 & 1528.20 & 105.42 & 98.95 & 83.65 \\
Estimates (emergency data set) & -144.06 & 1255.06 & 105.24 & 97.98 & 95.15 \\
\hline
\end{tabular}

Table 1

Comparison of the measured and estimated values using empirical RST parameters (nSv/h)

\begin{tabular}{|lrrrrr|}
\hline N = 808 & min. & max. & mean & median & std. dev. \\
\hline Observed (routine data set) & 57.00 & 180.00 & 98.02 & 98.80 & 20.01 \\
Estimates (routine data set) & 68.34 & 124.53 & 96.75 & 99.15 & 14.45 \\
\hline Observed (emergency data set) & 57.00 & 1528.20 & 105.42 & 98.95 & 83.65 \\
Estimates (emergency data set) & 66.83 & 257.09 & 109.71 & 99.78 & 39.70 \\
\hline
\end{tabular}

Table 2

Comparison of the measured and estimated values using parameters found by cross-validation (nSv/h)

The interpolation accuracy was analyzed using the mean absolute error (MAE), the mean error (ME), and the root mean squared error (RMSE) of the predictions at the 808 evaluation locations. These error characteristics are defined as follows:

$$
\begin{gathered}
M A E=\frac{1}{n} \sum_{i=1}^{n}\left|a_{i}^{*}-a_{i}\right|, \\
M E=\frac{1}{n} \sum_{i=1}^{n}\left(a_{i}^{*}-a_{i}\right), \\
R M S E=\sqrt{\frac{1}{n} \sum_{i=1}^{n}\left(a_{i}^{*}-a_{i}\right)^{2}},
\end{gathered}
$$


where $a_{i}^{*}$ is the estimated value at location $\mathrm{i}$ and $a_{i}$ is the true value. The Pearson's correlation coefficient $r$ for estimated and observed values was also computed.

The comparison of interpolation errors expressed by statistical characteristics (6) - (8) showed that parameters found by CV provided better overall results than empirical parameters (Table 4 and 5), except for ME. This clearly contradicts with the results in Table 1 and 2, where empirical parameters performed better. Therefore we decided to verify the optimal parameters using the evaluation data set released after the test phase. We iteratively computed estimates for 808 evaluation locations using the RST interpolation of both test data sets with parameters within a reasonable range (tension $\varphi \in<10,200>$, and smoothing $w \in<0.1,2.0>$ ) in order to find a combination with minimized interpolation errors (represented by RMSE and MAE). The optimal parameter combinations minimizing the interpolation error were tension $=200$, smoothing $=1.2$ for the routine data set and tension $=150$, smoothing $=1.2$ for the emergency data set. These parameters are different than the parameters found by $\mathrm{CV}$ (tension $=10$, smoothing $=0.6$ and tension $=10$, smoothing $=1.6$, respectively) and empirical parameters used in the test (tension $=40$, smoothing $=0.1$ ). The comparison of interpolation error statistics in Table 5 and 6 showed that $\mathrm{CV}$ did not find the optimal parameters with minimal prediction errors for both data sets.

The general statistics for the results obtained using these verified parameters are shown in Table 3. Note, that these "optimal" results were obtained via minimization of the overall prediction error defined by RMSE and MAE, and were not focused on preserving the anomalies in input data. Therefore the general statistics in Table 3 are only slightly better than the statistics for interpolation with parameters found by CV (Table 2) and still worse than the results obtained by interpolation with empirical parameters (Table 1). It should be noted again that the verified parameters were obtained after the test phase and these interpolation results are not part of the competition.

\begin{tabular}{|lrrrrr|}
\hline $\mathbf{N}=\mathbf{8 0 8}$ & min. & max. & mean & median & std. dev. \\
\hline Observed (routine data set) & 57.00 & 180.00 & 98.02 & 98.80 & 20.01 \\
Estimates (routine data set) & 68.23 & 131.63 & 96.72 & 99.30 & 14.33 \\
\hline Observed (emergency data set) & 57.00 & 1528.20 & 105.42 & 98.95 & 83.65 \\
Estimates (emergency data set) & 67.49 & 681.90 & 105.82 & 100.50 & 56.88 \\
\hline
\end{tabular}

Table 3

Comparison of the measured and estimated values using parameters found by verification $(\mathrm{nSv} / \mathrm{h})$

\begin{tabular}{|lrrrr|}
\hline Data sets & MAE & ME & Pearson's $\boldsymbol{r}$ & RMSE \\
\hline Routine data set & 10.28 & -1.11 & 0.73 & 14.00 \\
Emergency data set & 28.35 & 0.18 & 0.38 & 100.13 \\
\hline
\end{tabular}

Table 4

Comparison of the errors for interpolation with empirical parameters 


\begin{tabular}{|lrrrr|}
\hline Data sets & MAE & ME & Pearson's $\boldsymbol{r}$ & RMSE \\
\hline Routine data set & 9.38 & -1.27 & 0.78 & 12.68 \\
Emergency data set & 26.52 & 4.29 & 0.38 & 77.98 \\
\hline
\end{tabular}

Table 5

Comparison of the errors for interpolation with parameters found by $\mathrm{CV}$

\begin{tabular}{|lrrrr|}
\hline Data sets & MAE & ME & Pearson's $\boldsymbol{r}$ & RMSE \\
\hline Routine data set & 9.10 & -1.30 & 0.79 & 12.51 \\
Emergency data set & 18.62 & 0.41 & 0.50 & 73.68 \\
\hline
\end{tabular}

Table 6

Comparison of the errors for interpolation with parameters found by verification

This verification analysis has shown that the RST method cannot be parametrized by $\mathrm{CV}$ for low-density data if one of the interpolation goals is to detect anomalies and fluctuations in data. Also, it has been shown that the RST method with empirical parameters (tension=40 and smoothing=0.1) produced interpolation results that are more appropriate for detection of anomalies and outliers.

The spatial distribution of interpolated values can be seen on $2 \mathrm{D}$ and $3 \mathrm{D}$ maps (Figures 1-5). The 2D maps are shown with greyscale intervals of $10 \mathrm{nSv} / \mathrm{h}$ separated by white isolines. The cross symbols point to the locations of the estimated values; empty squares were used to indicate the locations of the input values.

Despite the fact that even verified parameters cannot be considered as the true image of the spatial radioactivity distribution, the RST interpolation with verified parameters shows the best result in terms of minimized overall prediction error that can be obtained by RST and test data sets. The comparison of Figures 1-2 for the routine data set and Figures 3-4 for the emergency data set clearly shows that parameters suggested by $\mathrm{CV}$ produced very smoothed results neglecting local fluctuations in data. The empirical parameters with lower tension (40) allowed a higher spatial influence of single points and therefore the spatial changes in estimated radioactivity are more graduate than in surfaces produced by RST with verified parameters. Even more striking are 3D maps showing interpolated surfaces using these 3 different sets of parameters in Figure 5a-c. As we mentioned above, the RST interpolation with empirical parameters for the second dataset produced negative radioactivity estimates that are visible in Figure $5 \mathrm{~b}$ as undershoots around the local spike in data values. The empirical parameters were chosen using training datasets that are similar to the routine test data set. The undershoots in the emergency test dataset can be minimized by a higher tension that reduces the influence of input points and the resulting surface behaves like a mebrane (Figure 5c). 

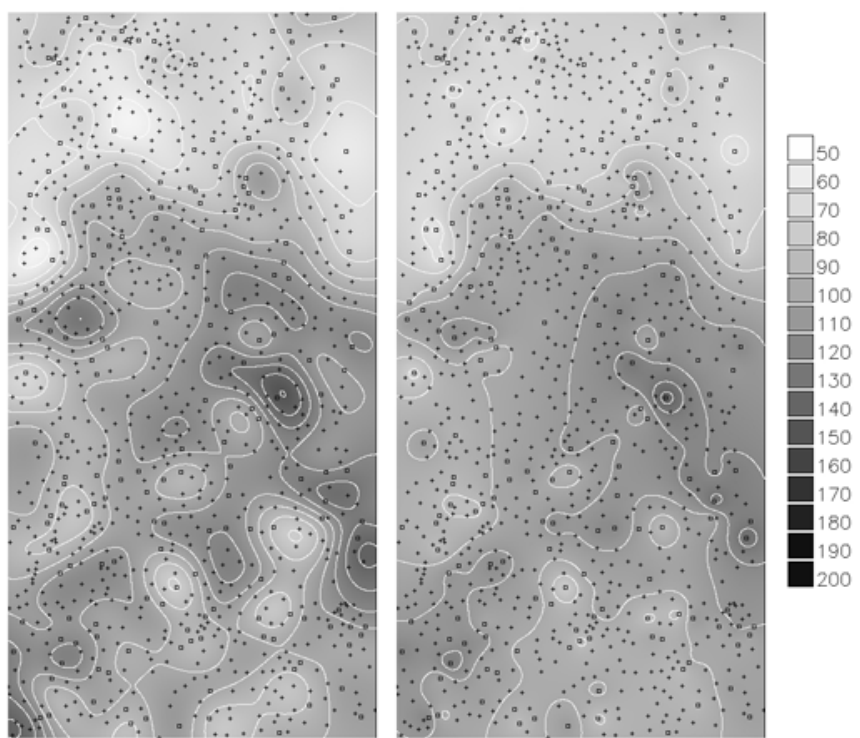

Figure 1

Interpolation of radioactivity with empirical parameters (left) and verified parameters (right) for the routine data set (nSv/h)
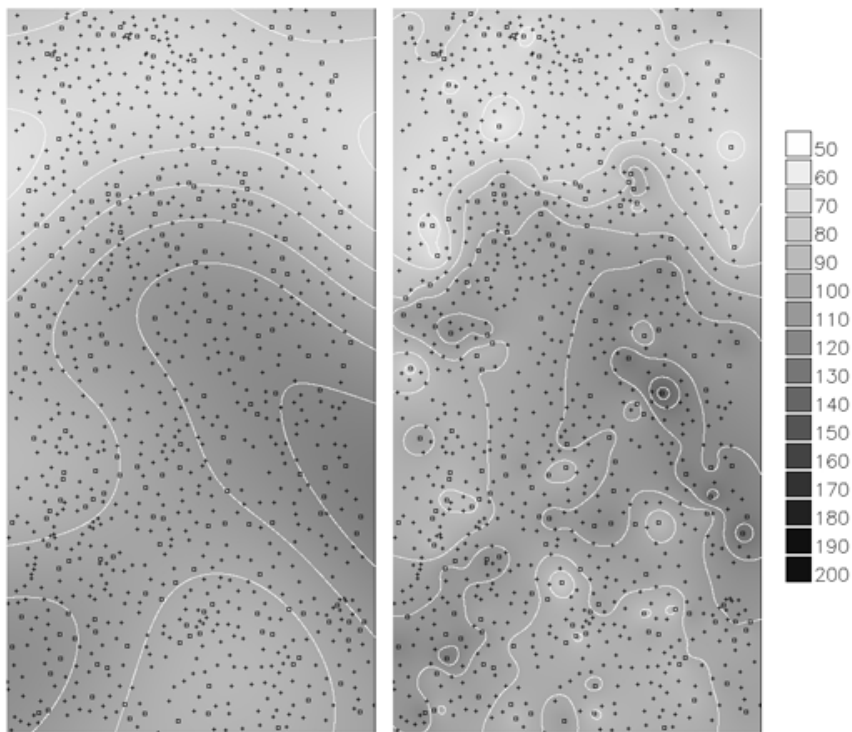

Figure 2

Interpolation of radioactivity with parameters found by cross-validation (left) and verified parameters (right) for the routine data set (nSv/h) 

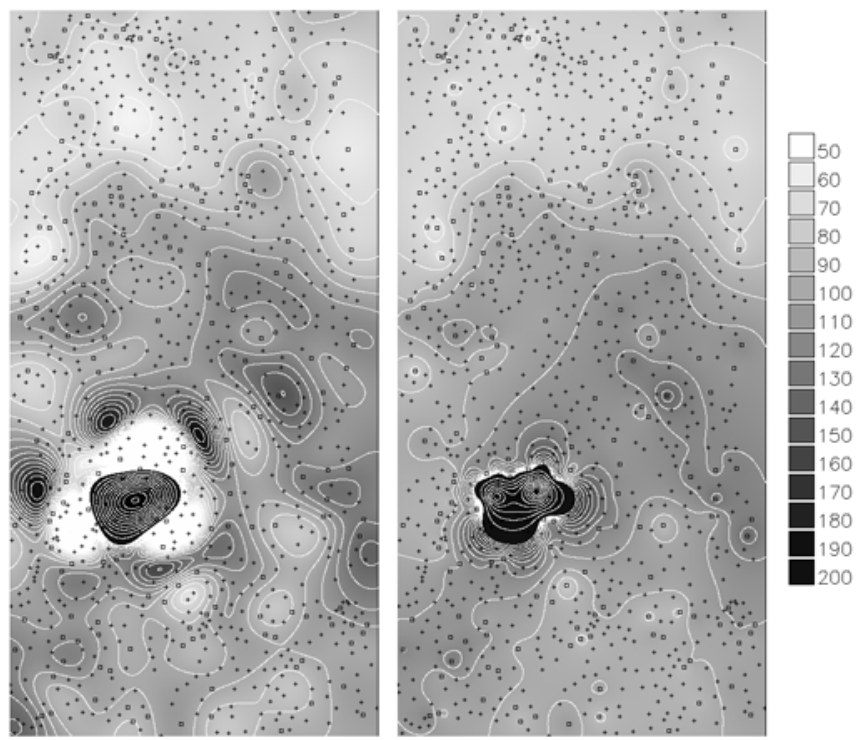

Figure 3

Interpolation of radioactivity with empirical parameters (left) and verified parameters (right) for the emergency data set (nSv/h)
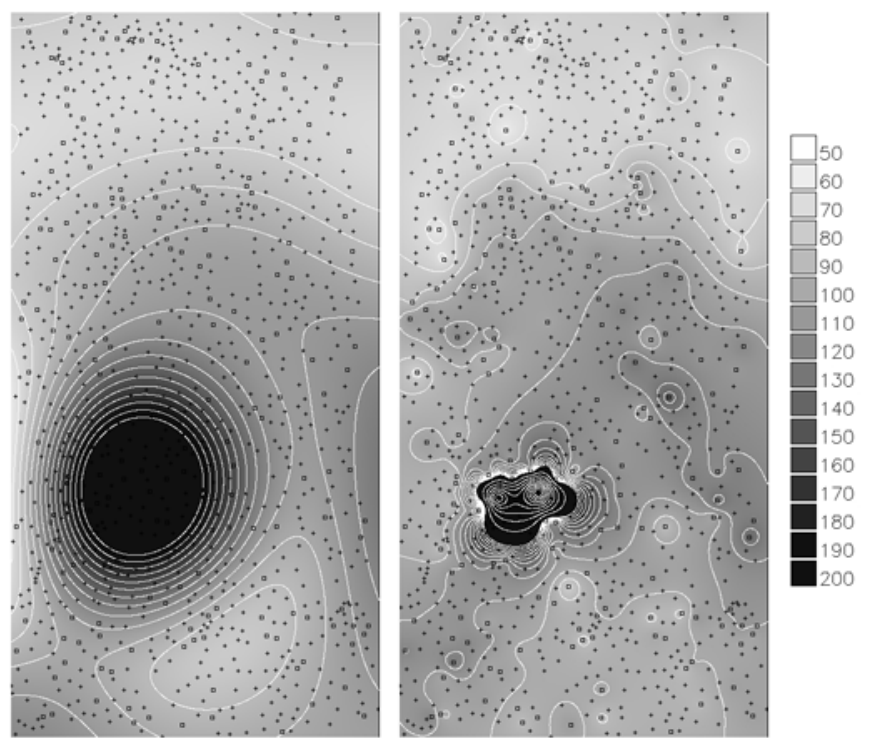

Figure 4

Interpolation of radioactivity with parameters found by cross-validation (left) and verified parameters (right) for the emergency data set (nSv/h) 

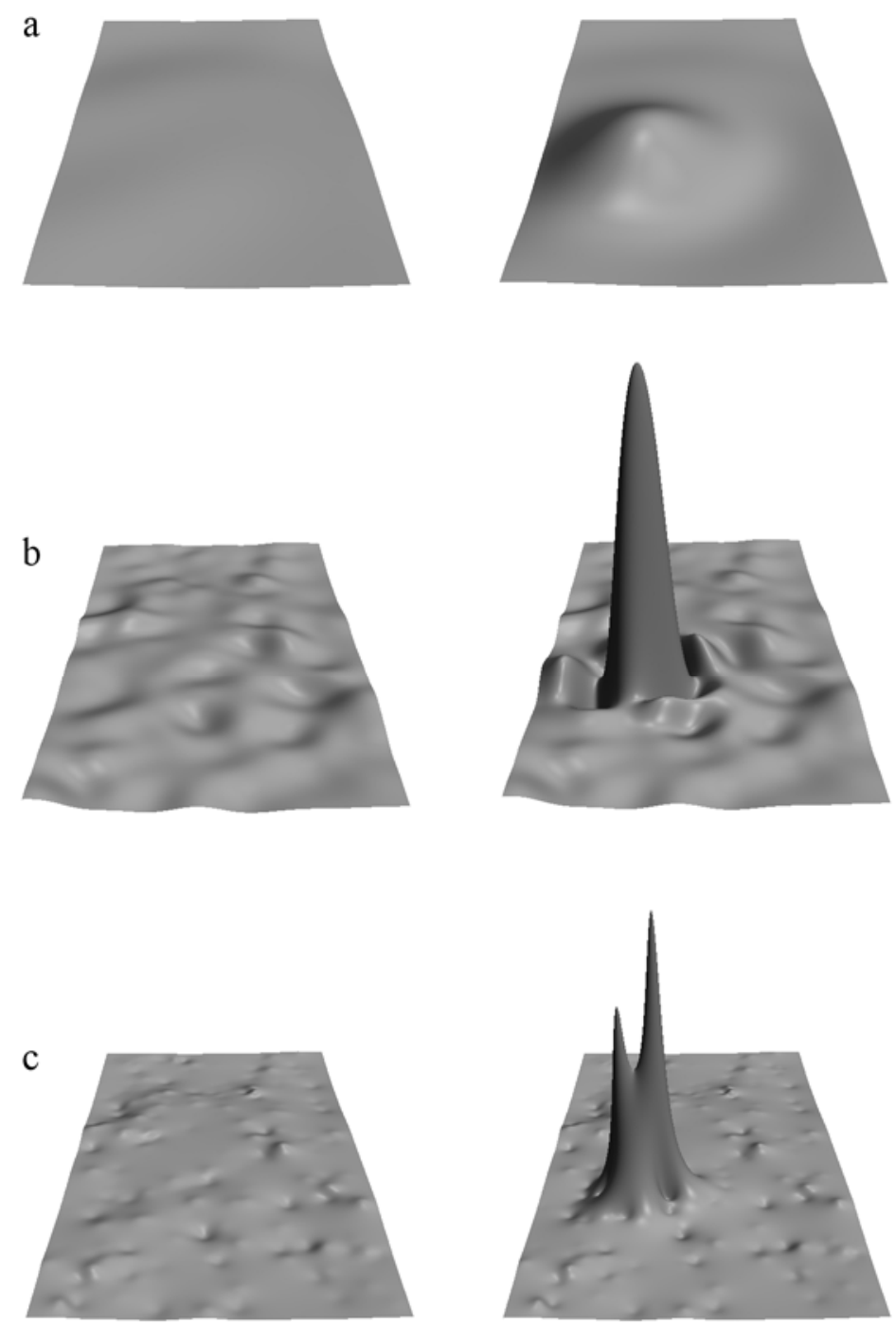

Figure 5

3D map showing interpolated surfaces using parameters found by a) a CV procedure, b) empirical estimation, c) verification for the routine (left) and emergency (right) data set

The application of CV to the optimization of RST parameters for radioactivity modeling showed the limits of this optimization technique in areas where input data are not sufficiently sampled. Anomalies and local fluctuation require a representative sampling especially in automatic mapping applications. Also, it has been shown that the RST parameters are flexible enough to produce interpolation results that reflects the behavior of the modeled phenomenon even for less dense data sets. However, the question of automatic parametrization is still open and depends heavily on available data and the purpose of spatial interpolation. This study also showed that the minimization of the overall 
interpolation error can be in contradiction to the detection of anomalies and local fluctuations.

The use of RST in automatic mapping and decision support systems are quite strighforward, as the method is deterministic, controled by a set of parameters that can be set manually or by optimization technique. Thus the user can use optimized or default parameters without in-depth knowledge of the method. On the other hand, the RST parameters are flexible enough to control the interpolation behavior according to the character of the modeled phenomenon. This study showed a great flexibility and robustness of this method.

The average computational time for both test data sets was about 40 seconds on a standard personal computer with 2.67 GHz CPU and Linux operating system. An open source environment of Linux and GRASS GIS enable modification and incorporation of the source code into wider systems focused, for example, on monitoring, automatic mapping and decision support.

\section{CONCLUSIONS}

In this paper we presented Regularized Spline with Tension and its application to radioactivity modeling within SIC2004 (see Dubois and Galmarini 2005). The results questioned the reliability of $\mathrm{CV}$ as an optimization technique in case of poorly sampled phenomena. The RST method applied to the provided data sets and parametrized by CV produced very smoothed interpolation results that were not appropriate for detecting radioactivity anomalies and emergency purposes. On the other hand, the flexibility and robustness of the RST method enable the parametrization by other methods that can deliver results reflecting anomalies and local fluctuations even in less dense data. However, the sampling density still remains a major problem that must be handled carefully prior to the application of any interpolation and parameter optimization method.

One of the advantages of the method is its flexibility within the single radial basis function. In addition, the method is applicable to both very large and small data sets. The method is deterministic and can be fully automated. It can be applied to a wide range of environmental variables with a trend or anisotropic behavior (Mitas and Mitasova 1999), (Hofierka et al. 2002). The source code of the presented method is available within an open source environment of GRASS GIS (Neteler and Mitasova 2002).

\section{REFERENCES}

Abramowitz, M; Stegun, IA. Handbook of Mathematical Functions. Dover, New York: 1964. Burrough, PA. Principles of Geographical Information Systems for Land Resources Assessment. Oxford University Press; 1986.

Cressie, NAC. Statistics for Spatial Data. New York: John Wiley \& Sons Inc.; 1993.

Dubois, G; Galmarini, S. 'Introduction to the Spatial Interpolation Comparison (SIC) 2004 exercise and presentation of the data sets'. Applied GIS; 1 (2): 09-01 to 09-11.

Duchon, J. 'Interpolation des fonctions de deux variables suivant le principe de la flexion des plaques minces: R.A.I.R.O.'. Analysis Numerique 1976; 10: 5-12.

Franke, R. 'Thin plate spline with tension'. Computer Aided Geometrical Design 1985; 2: 87-95.

Hofierka, J; Parajka, J; Mitasova, H; Mitas, L. 'Multivariate interpolation of precipitation using regularized spline with tension'. Transactions in GIS 2002; 6 (2): 135-150. 
Jeffrey, SJ; Carter, JO; Moodie, KB; Beswick, AR. 'Using spatial interpolation to construct a comprehensive archive of Australian climate data'. Environmental Modelling \& Software 2001; 16: 309-330.

Mitas, L; Mitasova, H. 'Spatial Interpolation'. In: Longley, P; Goodchild, MF; Maguire, DJ; Rhind, DW, editors. Geographical Information Systems: Principles, Techniques, Management and Applications. GeoInformation International, Wiley; 1999. pp. 481-492.

Mitas, L; Mitasova, H. 'General variational approach to the interpolation problem'. Computers and Mathematics with Applications 1988; 16: 983-992.

Mitasova, H; Mitas, L; Brown, BM; Gerdes, DP; Kosinovsky, I. 'Modeling spatially and temporally distributed phenomena: New methods and tools for GRASS GIS'. International Journal of GIS 1995; 9 (4): 443-446.

Neteler, M; Mitasova, H. Open Source GIS: A GRASS GIS Approach. Kluwer Academic Publishers; 2002.

Cite this article as: Hofierka, Jaroslav. 'Interpolation of radioactivity data using regularized spline with tension'. Applied GIS, Vol 1, No 2, 2005. pp. 16-01 to 16-13. DOI: 10.2104/ag050016

Copyright $\odot 2005$ Jaroslav Hofierka 